\title{
LETTER
}

\section{Efficacy of early prone position for COVID-19 patients with severe hypoxia: a single-center prospective cohort study}

\author{
Xuefeng Zang ${ }^{1}$, Qian Wang ${ }^{3}$, Hua Zhou ${ }^{6 *}$, Sanhong Liu ${ }^{2,5^{*}}$ (D) and Xinying Xue ${ }^{4,7^{*}}$ on behalf of COVID-19 Early \\ Prone Position Study Group
}

(c) 2020 The Author(s)

\section{Dear Editor,}

With the global epidemic of COVID-19, as of July 8 , $2020,12,025,348$ people have been infected with $4.56 \%$ mortality [1]. Many COVID-19 patients died due to severe hypoxia $[2,3]$. It is particularly important to find a simple and effective way for COVID-19 patients' treatment. Recent studies reported that prone position was used to treat non-intubated COVID-19 patients and hypoxemic acute respiratory failure [4, 5]. However, the number of patients was small, the follow-up was short, clinical outcomes were not assessed in their study. In this study, we aimed to explore whether the early prone position can effectively improve severe hypoxia, CT imaging performance and survival prognosis of COVID-19 patients with severe hypoxia.

A total of 60 COVID-19 patients with severe hypoxia were enrolled from February 1, 2020 to April 30, 2020 (Fig. S1, Tables S1 and S2) (Chinese Clinical Trial Registry: ChiCTR2000033053). And 23 patients were taken early prone position and 37 patients were not. In prone

\footnotetext{
*Correspondence: zha01481@btch.edu.cn; liush@shanghaitech.edu.cn; xinyingxue2010@163.com

${ }^{2}$ Institute of Interdisciplinary Integrative Medicine Research, Shanghai University of Traditional Chinese Medicine, Shanghai, China

${ }^{4}$ Department of Respiratory and Critical Care Medicine, Beijing Shijitan Hospital, Capital Medical University, Beijing, China

${ }^{6}$ Department of Critical Care Medicine, Beijing Tsinghua Changgung Hospital, School of Clinical Medicine, Tsinghua University, Beijing, China Full author information is available at the end of the article

Xuefeng Zang and Qian Wang contributed equally to this work. Hua Zhou, Sanhong Liu and Xinying Xue contributed equally to this work.

The members of the COVID-19 Early Prone Position Study Group are listed in the Acknowledgements.
}

position group, the pulse oxygen saturation $\left(\mathrm{SpO}_{2}\right)$ increased from $91.09 \pm 1.54 \%$ to $95.30 \pm 1.72 \%(P<0.01)$ after $10 \mathrm{~min}, 95.48 \pm 1.73 \%$ after $30 \mathrm{~min}(P<0.01)$, but no significant difference after $30 \mathrm{~min}$ compared with $10 \mathrm{~min}(P=0.58)$ (Fig. 1a). The respiratory rate (RR) decreased from $28.22 \pm 3.06$ times/min to $27.78 \pm 2.75$ times/min after $10 \mathrm{~min}(P=0.20), 24.87 \pm 1.84$ times/ min after $30 \mathrm{~min}(P<0.01)$, but no significant difference after 10 min compared with the baseline value $(P=0.203)$ (Fig. 1b). ROX index increased from $3.35 \pm 0.46$ after $10 \mathrm{~min}$ to $3.55 \pm 0.47(P<0.01), 3.96 \pm 0.45$ after $30 \mathrm{~min}$ $(P<0.01)$ (Fig. 1c). However, there was no significant difference in $\mathrm{SpO}_{2}$, RR and ROX index in a non-prone position group (Fig. 1d-i). Additionally, there is significant difference in $\mathrm{SpO}_{2}-10 \mathrm{~min}, \mathrm{ROX}-10 \mathrm{~min}, \mathrm{SpO}_{2}-30 \mathrm{~min}$, RR-30 min and ROX index-30 min between the two groups $(P<0.01)$ (Table $\mathrm{S} 3)$. Furthermore, the early prone position can also improve the $\mathrm{CT}$ imaging performance in some patients (Fig. 1j). After 90 days of follow-up, 10 (43.5\%) COVID-19 patients died in the prone position group, compared with 28 (75.7\%) COVID-19 patients in the non-prone position group (Fig. 1k). As for the potential mechanism of early prone position improving the hypoxia, we speculate that it may be caused by redistribution of blood flow and edema fluid redistributes to the ventral side with gravity and the atrophic alveolar are reopened in the prone position, which cause V/Q improvement.

There are also some limitations in our study: (1) Limited to the conditions at that time, some COVID-19 patients with severe hypoxia between two groups cannot be managed by the same researcher. (2) Limited to COVID-19 outbreak, blood gas determining respiratory 


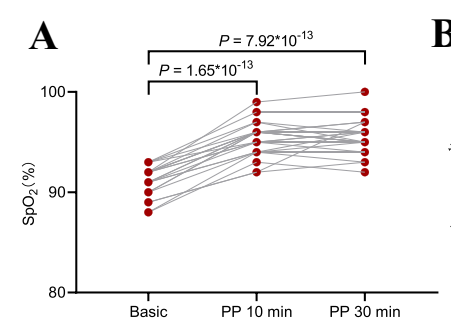

\section{B}

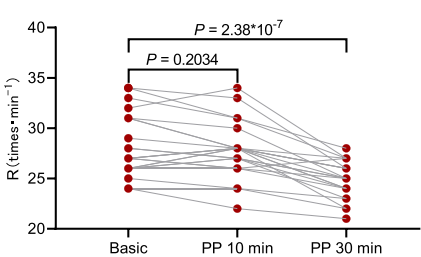

$\mathbf{E}$
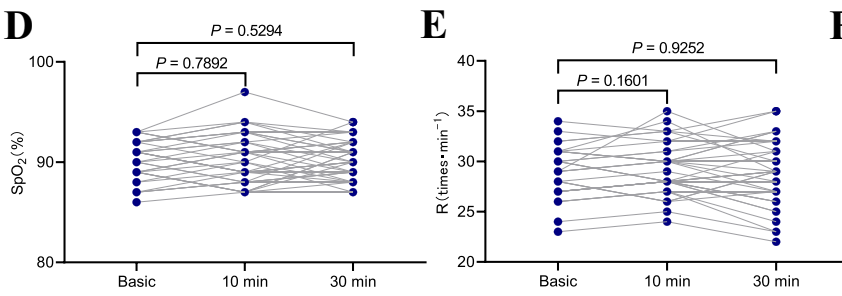

$\mathbf{F}$

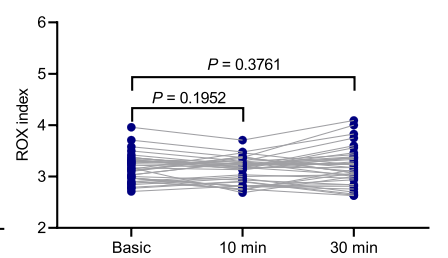

G
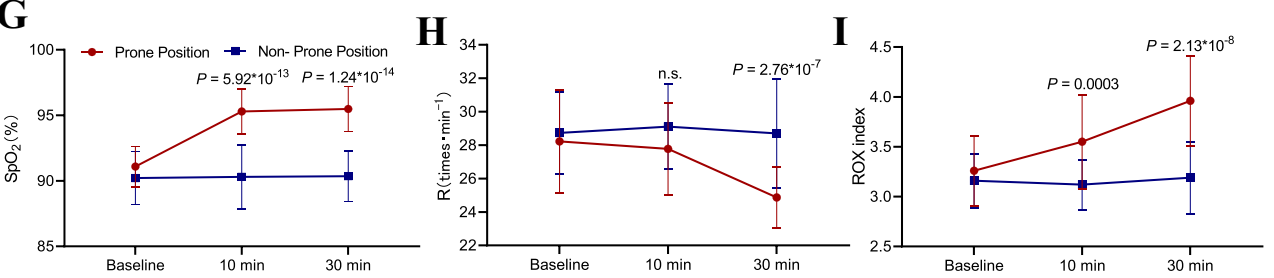
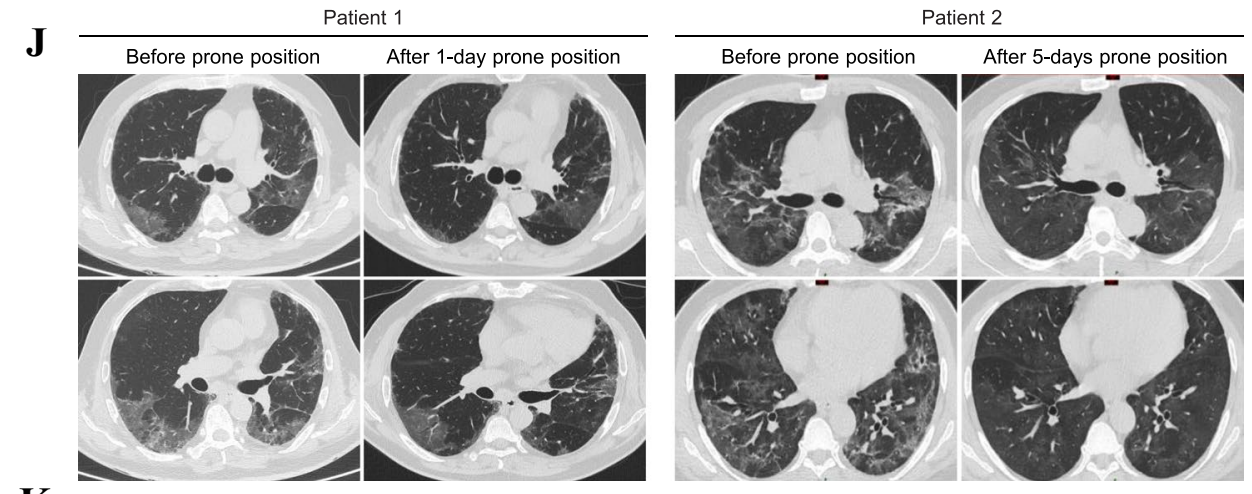

K

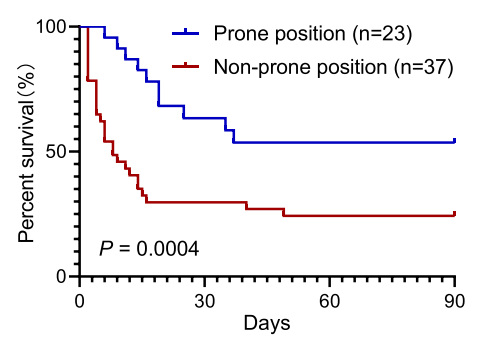

Fig. 1 Early prone position significantly improves $\mathrm{SpO}_{2}, \mathrm{RR}, \mathrm{ROX}$ index, $\mathrm{CT}$ imaging performance and reduce the mortality of COVID-19 patients with severe hypoxia. a-c The single $\mathrm{SpO}_{2}(\mathbf{a}), \mathrm{RR}(\mathbf{b})$, and ROX index (c) change in the prone position group. $\mathbf{d}-\mathbf{f}$ The single $\mathrm{SpO}_{2}(\mathbf{d}), \mathrm{RR}(\mathbf{e})$, and ROX index (f) change in the non-prone position group. $\mathbf{g}$-i The average $\mathrm{SpO}_{2}(\mathbf{g})$, average RR (h), and average ROX index (i) change between prone position and non-prone position groups. $\mathbf{j}$ In patient 1, CT imaging showed that the density and scope of diffuse patch shadow in both lungs was significantly improved after 1-day prone position. In patient 2, CT imaging showed that the patch shadow was completely absorbed after 5-day prone position. $\mathbf{k}$ Survival curve of COVID-19 patients with severe hypoxia between prone position group and non-prone position group 
failure cannot be analyzed in time. (3) The number of COVID-19 patients with severe hypoxia enrolled in this study is also limited.

In conclusion, this work will have value in helping clinicians to use early prone position to treat COVID-19 patients with severe hypoxia, it may reduce the mortality of COVID-19 patients with severe hypoxia, and help guide appropriate and effective management for future patients.

\section{Electronic supplementary materia}

The online version of this article (https://doi.org/10.1007/s00134-020-06182-4) contains supplementary material, which is available to authorized users.

\begin{abstract}
Author details
${ }^{1}$ Department of Critical Care Medicine, Beijing Shijitan Hospital, Capital Medical University, Beijing, China. ${ }^{2}$ Institute of Interdisciplinary Integrative Medicine Research, Shanghai University of Traditional Chinese Medicine, Shanghai, China. ${ }^{3}$ Department of Geriatrics, Union Hospital, Tongji Medical College, Huazhong University of Science and Technology, Wuhan, China. ${ }^{4}$ Department of Respiratory and Critical Care Medicine, Beijing Shijitan Hospital, Capital Medical University, Beijing, China. ${ }^{5}$ Shanghai Institute for Advanced Immunochemical Studies, ShanghaiTech University, Shanghai, China. ${ }^{6}$ Department of Critical Care Medicine, Beijing Tsinghua Changgung Hospital, School of Clinical Medicine, Tsinghua University, Beijing, China. ${ }^{7}$ Hospital of Weifang Medical University, Weifang, People's Republic of China.
\end{abstract}

\section{Acknowledgements}

We would like to thank the COVID-19 Early Prone Position Study Group members for their efforts: Weidong Zhang, Institute of Interdisciplinary Integrative Medicine Research, Shanghai University of Traditional Chinese Medicine, Shanghai, China. Quansheng Gao, Department of Operational Medicine Tianjin Institute of Environmental \& Operational Medicine, Tianjin, China. Jiang Xie, Department of Respiratory and Critical Care Medicine, Beijing Anzhen Hospital, Capital Medical University, Beijing, China. Chunguo Jiang, Department of Respiratory and Critical Care Medicine, Beijing Chaoyang Hospital, Capital Medical University, Beijing, China. Xinmin Ding, Jie Zhang, Department of Respiratory and Critical Care Medicine, Beijing Shijitan Hospital, Capital Medical University, Beijing, China. Feiping Lu, Wei Chen, Jie Zhen, Department of Critical Care Medicine, Beijing Shijitan Hospital, Capital Medical University, Beijing, China. Ping He, Meng'en Zhu, Department of Geriatrics, Union Hospital, Tongji Medical College, Huazhong University of Science and Technology, Wuhan, China. Xiaomeng Zhang, Department of Otolaryngologic Surgery, Union Hospital, Tongji Medical College, Huazhong University of Science and Technology, Wuhan, China. Xiaodong Yuan, Department of Infectious Disease, Beijing Shijitan Hospital, Capital Medical University, Beijing, China. Rong Tian, Department of Respiratory, Beijing Geriatric Hospital, Beijing, China. Xiaohui Wang, Department of Infectious Diseases, Beijing Tsinghua Changgung Hospital, School of Clinical Medicine, Tsinghua University, Beijing, China. Nan Zhang, Department of Critical Care Medicine, Beijing Chest Hospital, Capital Medical University, Beijing, China. Jun Guo, Department of Pulmonary and Critical Care Medicine, Beijing Tsinghua Changgung Hospital, School of Clinical Medicine, Tsinghua University, Beijing, China. Xuelei Zang, Center of Clinical Laboratory Medicine, the first Medical Centre, Chinese PLA General Hospital, Beijing, China. Xidong Ma, Department of Respiratory and Critical Care, the first Medical Centre, Chinese PLA General Hospital, Beijing, China. Zhiqiang Xue, Department of Chest surgery, Chinese PLA General Hospital, Beijing, China.

\section{Author contributions}

$X Z, Q W, H Z, S L$, and XX conceptualized the article. XZ, QW, and XX analyzed the data. $X Z, Q W, H Z, S L$, and $X X$ wrote the initial draft. $H Z, S L$, and $X X$ are the guarantors.

\section{Funding}

National Natural Science Fund Youth Project (81700007); National Natural Science Foundation of China (81772798, 62041602); Research and innovation fund of the Ministry of Education (2018A03026); Beijing Natural Science Foundation (2019A10); "Qingmiao" plan of Beijing Municipal Hospital Administration (2018QM4); Outstanding top talent (2019YXBJ1); Capital health development scientific research unit matching fund (2020-2Z-2086). The National Key Research and Development Program of China (2020YFC0845400 \& 2017YFC1700200); Excellent talents in Beijing "Youth top team" (2019YXBJ2); Capital health development scientific research unit matching fund (2020-2Z-2086)

\section{Compliance with ethical standards}

\section{Conflicts of interest}

The authors have no conflicts of interest to disclose.

\section{Open Access}

This article is licensed under a Creative Commons Attribution-NonCommercial 4.0 International License, which permits any non-commercial use, sharing, adaptation, distribution and reproduction in any medium or format, as long as you give appropriate credit to the original author(s) and the source, provide a link to the Creative Commons licence, and indicate if changes were made. The images or other third party material in this article are included in the article's Creative Commons licence, unless indicated otherwise in a credit line to the material. If material is not included in the article's Creative Commons licence and your intended use is not permitted by statutory regulation or exceeds the permitted use, you will need to obtain permission directly from the copyright holder. To view a copy of this licence, visit http://creativecommons.org/licen ses/by-nc/4.0/.

\section{Publisher's Note}

Springer Nature remains neutral with regard to jurisdictional claims in published maps and institutional affiliations.

Accepted: 11 July 2020

Published online: 22 July 2020

\section{References}

1. Worldometers. COVID-19 CORONAVIRUS PANDEMIC. 2020-7-8]. https:// www.worldometers.info/coronavirus/\#coronavirus-cases-log.

2. Ruan Q et al (2020) Clinical predictors of mortality due to COVID-19 based on an analysis of data of 150 patients from Wuhan, China. Intensive Care Med 46(5):846-848

3. Ruan Q et al (2020) Correction to: Clinical predictors of mortality due to COVID-19 based on an analysis of data of 150 patients from Wuhan, China. Intensive Care Med 46(6):1294-1297

4. Elharrar X et al (2020) Use of prone positioning in nonintubated patients with COVID-19 and hypoxemic acute respiratory failure. Jama 323(22):2336-2338

5. Sartini $\mathrm{C}$ et al (2020) Respiratory parameters in patients with COVID-19 after using noninvasive ventilation in the prone position outside the intensive care unit. Jama 323(22):2338-2340 